\title{
Management of Edentulous Resorbed Mandibular Residual Ridge in Malaysian Geriatric Patients: 2-Year Clinical Study
}

\section{ABSTRACT}

Objectives: A combination of the neutral zone and the polished surface impression techniques is a useful method to reserve the functional and physiological forms of the underlying mandibular residual ridge by enhancing both retention and stability in Malaysian geriatric patients (2-year clinical study).

Materials and methods: A total of 25 Malaysian geriatric patients have participated in the current study. Two sets of dentures were served to the patients. The first set was constructed according the conventional method of complete denture construction whereas the second set was constructed by using neutral zone and polished surface impression techniques.

Results: The patients were followed up for 2 years and the obtained results showed that all patients have stopped using the conventional dentures and continued with the test dentures. The comparative analysis exhibited significant difference $(p<0.05)$ in favor of test dentures.

Conclusion: Combination of the neutral zone and the polished surface techniques can impart a physiological reservation to the underlying residual ridge without changes.

Keywords: Neutral zone, Polished surface impression, Stability and retention, Resorbed residual ridge, Complete denture, Geriatric patients.

Statement of problem: It is common for completely edentulous geriatric patients to have resorbed mandibular residual ridge because of the process of aging. Some surgical interactions are contraindicated for this age group of patients, so, neutral zone technique and/or polished surface impression verified effective in improving stability for lower complete dentures. In spite of the fact that the above mentioned methods often used to enhance stability of complete denture, most of the studies were relying on only one of those methods to improve stability rather than retention. Researcher hypothesis stated that a combination of the neutral zone and the polished surface impression techniques should be performed together to enhance both stability and retention and henceforth reservation of the residual ridge under mandibular complete dentures issued to the patients with a constant follow-up for two consecutive years.

How to cite this article: Mustafa AA. Management of Edentulous Resorbed Mandibular Residual Ridge in Malaysian Geriatric Patients: 2-Year Clinical Study. Int J Prosthodont Restor Dent 2013;3(2):83-86.

Source of support: Nil

Conflict of interest: None

\section{INTRODUCTION}

Residual ridge resorption is a common sequel for edentulism of the lower arch. This problem is aggravated with geriatric patients, ${ }^{1}$ so that, loose and unstable lower complete denture is a major problem confronted by denture patients. One of the methods used to solve this problem is to reshape the polished surfaces of the mandibular complete denture to register the shape of the surrounding muscles which is first described by Sir Wilfred Fish who has described a denture as having three surfaces with each surface playing an independent and pivotal role in the overall fit, stability, and comfort of the denture. Sir Wilfred has mentioned a dead zone to be registered during teeth arrangement which, henceforward, known as the neutral zone. ${ }^{2}$ Neutral zone technique is another solution for resorbed mandibular residual ridge. It is defined as the potential space between lips and cheeks on one side and tongue on the other side; that area or position of teeth where the forces between tongue and cheeks or lips are equal. ${ }^{3}$ Not malpositioned natural teeth are situated on this zone where, at the same time, the artificial teeth should be set exactly to equalize the forces selected by tongue and cheeks or lips. The neutral zone philosophy is based on a theory that for every force there is a reaction equal in the amount and opposite in the direction. In complete denture patients, this concept is understood as for every single patient, there is a definite area where the effect of the surrounding muscles will not dislodge the denture from its place and where lateral and forward forces produced by the tongue are counteracted by their opposite forces produced by the lips and cheeks. ${ }^{4}$ Proper accomplishment of this zone by proper handling of the steps of the neutral zone technique has a great impact on the success of removable prostheses constructed for the treatment of resorbed mandibular residual ridge. ${ }^{5,6}$ This achievement should be maintained well by full understanding to the philosophy of this technique. Since the objective of a complete denture is to restore the normal function and health of the patient, the operator should consider the proper techniques when constructing the prostheses.

\section{MATERIALS AND METHODS}

A total of 25 patients were selected (Table 1) with an average age range of 78.5 years from outpatients' dental clinic, Faculty of Dentistry, International Islamic University, Malaysia. Ethical approval was obtained from the ethical committee of the University. The patients have signed a consent form for participation in the study. All patients 


\begin{tabular}{lccc}
\multicolumn{5}{c}{ Table 1: Distribution of age, gender and ethnicity of patients } \\
\hline Gender & Number of patients & Age (Average range) & Ethnicity \\
\hline Females & 10 & 76.4 & 5 Indians \\
& & & 3 Chinese \\
& 15 & 80.8 & 2 Malaysian \\
Males & & & 5 Malaysian \\
& & 5 Indians \\
Total & 25 & 5 Chinese & 25
\end{tabular}

presented with bone resorption in the lower jaw ranging from moderate to severe. Orthopantomogram (OPG) (Promax 3D, Planmeca Oy, Helsinki Finland) was used in addition to clinical investigations to determine the severity of bone resorption (Fig. 1). Bone resorption was assessed depending on a classification system for complete edentulism by McGarry et al (1999). ${ }^{7}$ The main chief complain of those patients was unsatisfactory lower complete denture because of poor stability and retention. A preliminary impression was made, and the two sets of special trays were constructed on the same study cast. Final impression, vertical dimension and centric jaw relation were carried out according to the usual conventional techniques.

A neutral zone technique was performed to the test denture after the registration of the maxillomandibular records. A continuous wire spurs $(1.20 \mathrm{~mm} / 47$ Dentaurum; Ispringen, Germany Lot: 406804) were attached to the record base (Fig. 2A) then a putty of heavy body hydrophilic vinyl siloxane impression material (Take-1, the hydrophilic vinyl type 0 very high consistency, Kerr; Germany Lot: 590184)



Fig. 1: OPG image showing an obvious mandibular ridge resorption in edentulous arch

were adjusted on the wire spurs (Fig. 2B). The neutral zone area was registered by asking the patients to make all the functional movements with lips, cheeks and tongue. Two sets of trial dentures were made for the try-in stage and both sets of dentures were examined for vertical dimension and centric relation verification, also for patient esthetic needs. Conventional set was carved by the technician. Then the following procedure was followed for the test dentures: the wax was removed from the buccal, labial and lingual
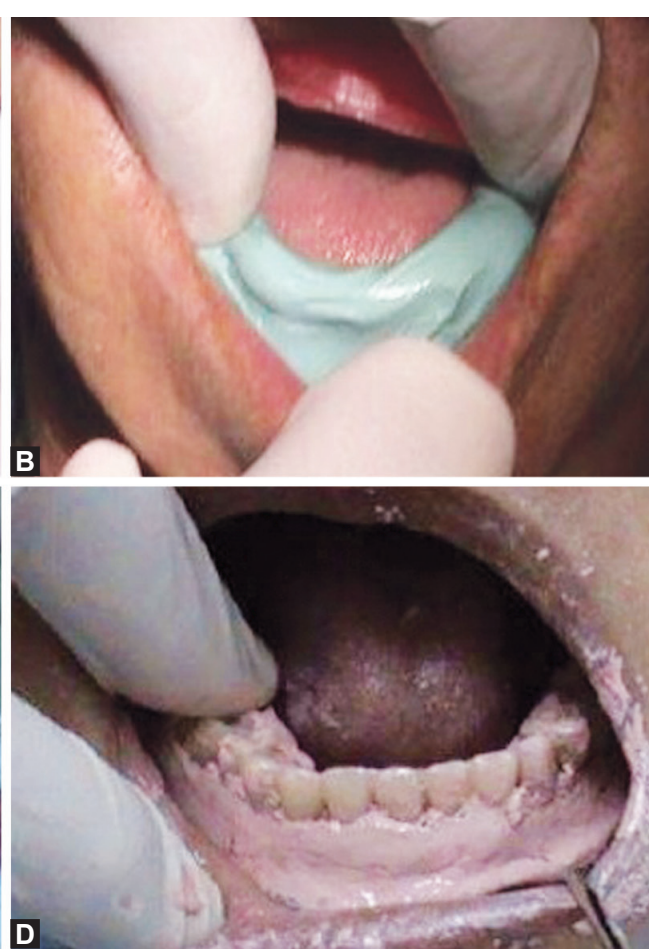

Figs 2A to D: Record base with wire spurs prior to neutral zone registration (A). Siloxane putty is then adjusted onto the wire spurs to make the registration (B). Removal of wax from buccal surface to provide a space for polished surface impression material (C). Making polished surface impression by using zinc oxide eugenol (D). 
surfaces to create a space for the impression material (Fig. 2C). The teeth were coated with Vaseline to ease the removal of the impression material that might cover it. Zinc oxide eugenol (ZOE) impression material (SS White, Gloucester England Lot: 841115) was used to register the function of lips, cheeks and tongue. ZOE was mixed and loaded first on the labial and buccal surfaces of the trial denture. With the upper trial denture in place, the lower denture was placed in the mouth (Fig. 2D), and the patient was asked to close in centric and was instructed to make normal movements of swallowing more than one time while ZOE was set to register the functional movement of the cheek and lower lip. When the material set, the trial denture removed from the mouth, and the excess material removed from the teeth. Same procedure was repeated for the lingual surface of the trial denture to register the action and movement of the tongue during swallowing. After cleaning, the lower trial denture was ready for flasking without any alteration in the impression surface. A copy cast was made by duplicating the master cast by using agar-agar impression material (Castogel BEGO, Bremen, Germany Lot: 1749) for the processing of the test denture. The technician was instructed not to overtrim these surfaces so that the anatomical land-mark at the lip, cheek and tongue as concavities and convexities were left unharmed. The conventional set was carved, processed and polished in the usual manner. The patients were given one set of the denture (conventional) to be used in the first month without knowing which set was issued first. Then, the test set was issued to the patients after 1 month. All those patients were asked for their evaluation for denture stability and retention, and this was confirmed through clinical evaluation by interexaminer evaluation (research personnel were not involved). An index by Hoad-Reddick and Grant was used to examine the retention and stability of the dentures. ${ }^{8}$ Qualitative and quantitative comparative analysis were conducted and then compared by crosstabulation. Significance between test and conventional dentures was measured by Chi-square test. p-value was set for significance to be $(p<0.05)$ and degree of freedom (df) $=1$. Number of follow-up visit with denture postinsertion complaints was recorded for each type.

\section{RESULTS}

Table 2 is showing the frequency of retention and stability values for both test and conventional dentures. Chi-square test shows a significant difference $(\mathrm{p}<0.05)$ between test and conventional dentures. The comparative analyses obtained by Hoad-Reddick and Grant showed that test dentures have exhibited higher numerical values of retention and stability when examined by interexaminer evaluation. The comparative analysis exhibited significant difference $(p<0.05)$ in favor of dentures constructed according to neutral zone and polished surface techniques with rooms for the muscles of lips, cheeks and tongue.

Table 2: Frequency of retention and stability values for both test and conventional dentures. Standard deviations and significance values are shown in Chi-square tests

Frequency of retention and stability of test dentures

\begin{tabular}{|c|c|c|c|c|c|c|}
\hline \multirow{5}{*}{ Valid } & & Frequency & Percentage & $S D$ & Valid percentage & Cumulative percentage \\
\hline & No & 0 & 00.0 & 0 & 00.0 & \multirow[t]{4}{*}{50.0} \\
\hline & Some & 6.0 & 24.0 & \pm 1.75 & 24.0 & \\
\hline & $\begin{array}{l}\text { Good and } \\
\text { sufficient }\end{array}$ & 19.0 & 76.0 & \pm 2.25 & 76.0 & \\
\hline & \multirow[t]{3}{*}{ Total } & 25 & 100.0 & & 100.0 & \\
\hline \multicolumn{6}{|c|}{ Frequency of retention and stability of conventional dentures } & \\
\hline & & Frequency & Percentage & $S D$ & Valid percentage & Cumulative percentage \\
\hline \multirow[t]{4}{*}{ Valid } & No & 13.0 & 52.0 & \pm 2.7 & 52.0 & 50.0 \\
\hline & Some & 10 & 40.0 & \pm 3.75 & 40.0 & 91.7 \\
\hline & $\begin{array}{l}\text { Good and } \\
\text { sufficient }\end{array}$ & 2 & 8.0 & \pm 3.25 & 8.0 & 100.0 \\
\hline & Total & 25.0 & 100.0 & & 100.0 & \\
\hline \multicolumn{7}{|c|}{ Chi-square tests } \\
\hline & & & Value & $d f$ & \multicolumn{2}{|c|}{ Asymp. sig. (2-sided) } \\
\hline \multicolumn{3}{|c|}{ Pearson Chi-square } & $6.000^{a}$ & 2 & \multicolumn{2}{|c|}{0.050} \\
\hline \multicolumn{3}{|c|}{ Likelihood ratio } & 7.638 & 2 & \multicolumn{2}{|c|}{0.022} \\
\hline \multicolumn{3}{|c|}{ Linear-by-linear association } & 4.568 & 1 & \multicolumn{2}{|c|}{0.033} \\
\hline \multicolumn{3}{|c|}{ McNemar-Bowker test } & 0.0 & 0.0 & \multicolumn{2}{|c|}{$0.0^{\mathrm{b}}$} \\
\hline \multicolumn{3}{|c|}{ No. of valid cases } & 25 & & & \\
\hline
\end{tabular}

${ }^{\mathrm{a}} 6$ cells $(100.0 \%)$ have expected count less than 5 . The minimum expected count is $0.33 ;{ }^{b}$ Computed only for a PXP table, where $p$ must be greater than 1 
On the other hand, the obtained records for denture postinsertion follow-up and adjustments showed that the conventional dentures needed more visits and adjustment procedures.

\section{DISCUSSION}

Loose and unstable mandibular complete dentures are common problems with resorbed lower residual ridge. ${ }^{9}$ After 2 years of following up the patients concerning the current study, it was extremely obvious that all the patients stopped using the conventional denture and they kept on using the test denture as the appliance of choice. All patients showed more comfort and faster accommodation to the test denture. On the other hand, the conventional dentures required more adjustment procedures that could be considered more time consuming in dental clinics. In addition to the above, patients suffered from ulceration and redness while they were using the conventional dentures, whereas there were no complaints about this matter during the use of the test dentures.

There were ample studies that have shown an increase in stability and retention with the use of neutral zone technique only, on the other hand other studies exhibited more retention and stability with using polished surface techniques. ${ }^{10-12}$ Infact, there is a significant correlation between the horizontal forces by lips, cheeks and tongue and the balanced placed of artificial teeth on dentures that can be achieved by neutral zone technique. ${ }^{13,14}$ It is very crucial to understand the patient's oral conditions and to link these findings to the treatment plan.

One can visualize that if the polished surface area is taken in terms of square unit area, the polished surface can be larger than impression and occlusal surfaces combined depending on anatomic structures. ${ }^{4}$ Therefore, in order for a denture to function properly, the contour of the polished surface of denture must be fabricated just as accurate as fit and contour of the impression and occlusal surfaces. By this, there will be a creation of room-like concavities for the surrounding muscles to sit inside these rooms. In addition to that, intimate contact with lip, cheeks, and tongue can be achieved and this will improve the stability of the denture in place.

\section{CONCLUSION}

A neutral zone technique should be performed in conjunction with polished surface impression for surrounding muscles to improve the retention and stability when constructing a mandibular complete denture for geriatric patients with a completely edentulous resorbed lower residual ridge. Consequently, and as shown by the obtained results, the test dentures were kept on serving the patients for
2 years with no major complaints. As a major sequel for the improvement of retention and stability, the ultimate benefit of this combination is to impart a physiological reservation to the underlying residual ridge without changes.

\section{ACKNOWLEDGMENTS}

The current study was funded by Research Management Center (RMC) International Islamic University Malaysia. The study was a part of research grant EDW B 0902-217 and also it was a fully covered by research grant EDW A 11-134-0925. The author would like to thank RMC for their kind support to the current study.

\section{REFERENCES}

1. Divaris K, Ntounis A, Marinis A, Polyzois G, Polychronopoulou A. Loss of natural dentition: multi-level effects among a geriatric population. Gerodontology 2012;29(2):192-199.

2. Fish EW. Using the muscles to stabilize the full lower denture. J Am Dent Assoc 1933;20:2163-2169.

3. The glossary of prosthodontics terms. J Prosthet Dent 2005;94:10-92.

4. Beresin VE, Schiesser FJ. The neutral zone in complete dentures. J Prosthet Dent 1976;36(4):356-367.

5. Weinberg L. Tooth position in relation to the denture base foundation. J Prosthet Dent 1958;8(3):398-405.

6. Lost PE. Fine arts in the fallacy of the ridges. J Prosthet Dent 1954;4(1):6-16.

7. McGarry TJ, Nimmo A, Skiba JF, Ahlstrom RH, Smith CR, Koumjian JH. Classification system for complete edentulism. The American College of Prosthodontics. J Prosthodont 1999;8(1):27-39.

8. Hoad-Reddick G, Grant AA. Prosthetic status: the formation of a schedule. J Prosthet Dent 1988;59(1):105.

9. Kokuto Y, Fukushimas S, Sato J, Seto K. Arrangement of artificial teeth in neutral zone after surgical reconstruction of mandible: a clinical report. J Prosthet Dent 2002; 88(2):125-127.

10. Fahmi FM. The position of the neutral zone in relation to the alveolar ridge. J Prosthet Dent 1992;67:805-809.

11. Alfano SG, Leupold RJ. Using neutral zone to obtain maxillomandibular relationship records for complete denture patients. J Prosthet Dent 2001;85(6):621-623.

12. Raybin NH. The polished surface of complete dentures. J Prosthet Dent 1963;13(2):236-239.

13. Frank J, Schiesser JR. The neutral zone and polished surfaces in complete dentures. J Prosthet Dent 1964;14(5):854-865.

14. Lynch CD, Allen PF. Overcoming the unstable mandibular complete denture: the neutral zone impression technique. Dent Update 2006;33(1):21-22, 24-26.

\section{ABOUT THE AUTHOR}

\section{Ammar A Mustafa}

Assistant Professor, Department of Prosthodontics and Dental Materials, Kulliyyah (Faculty) of Dentistry, International Islamic University Malaysia, Pahang, Malaysia, Phone: 0060199202975 e-mail: drammar71@gmail.com 